08

\title{
Исследование поведения структурных дефектов в нанокристаллах $\mathrm{CdSe}_{x} \mathrm{~S}_{1-x}$
}

\author{
(С) П.Г. Петросян, Л.Н. Григорян \\ Ереванский государственный университет, \\ 0025 Ереван, Армения \\ e-mail: ppetros@ysu.am
}

(Поступило в Редакцию 21 марта 2016 г. В окончательной редакции 12 сентября 2016 г.)

Представлены экспериментальные результаты, полученные при исследовании спектральной зависимости коэффициента поглощения нанокристаллов $\mathrm{CdSe}_{x} \mathrm{~S}_{1-x}$ в силикатном стекле с разной степенью совершенства кристаллической решетки. Получена зависимость концентрации дефектов от времени термообработки. Показано, что процесс исчезновения дефектов имеет тепловой активационный характер.

DOI: $10.21883 /$ JTF.2017.03.44252.1858

\section{Введение}

Полупроводниковые нанокристаллы интенсивно исследуются, так как они представляют научный и практический интерес. Физические свойства нанокристаллов сильно зависят от их размеров $[1-5]$. В полупроводниковых нанокристаллах семейства $\mathrm{A}^{\mathrm{II}} \mathrm{B}^{\mathrm{VI}}$ наблюдается яркая люминесценция в видимой области спектра, что позволяет применять их в биологических исследованиях, в производстве дисплеев, лазеров и газовых сенсоров [6-10].

Из семейства соединений $\mathrm{A}^{\mathrm{II}} \mathrm{B}^{\mathrm{VI}}$ важнейшими являются $\mathrm{CdS}$ и $\mathrm{CdSe}$, с помощью которых образуется полупроводниковое соединение $\mathrm{CdSe}_{x} \mathrm{~S}_{1-x}$. Оно имеет прямозонную структуру с перестраиваемой шириной запрещенной зоны в диапазоне от 1.7 до $2.4 \mathrm{eV}$. Оптические свойства нанокристаллов в основном обьясняются квантовым размерным эффектом [11,12]. Распределение дискретных уровней по энергии в нанокристаллах, определяющее оптические свойства таких объектов, зависит как от их размеров, так и от наличия в них структурных дефектов. Наличие структурных дефектов в нанокристаллах может существенно повлиять на периодическое распределение потенциала кристаллической решетки и стать решающим фактором в процессе формирования оптических свойств $[13,14]$.

Целью настоящей работы является исследование поведения структурных дефектов в нанокристаллах $\mathrm{CdSe}_{x} \mathrm{~S}_{1-x}$, а также их влияние на спектры оптического пропускания.

\section{Экспериментальные результаты и их обсуждение}

Исследуемые образцы были изготовлены по технологии, описанной в работе [13]. Образцы отличались друг от друга средними размерами, а также степенью совершенства кристаллической решетки нанокристаллов. Формирование нанокристаллов разных размеров и разной степени совершенства кристаллической решетки осуществлялось выбором температуры и длительности термообработки.

Были исследованы образцы двух серий. В образцах первой серии $T$ формирование полупроводниковых нанокристаллов в силикатном стекле осуществлялось при температуре $530^{\circ} \mathrm{C}$, а в образцах второй серии А - при температуре $500^{\circ} \mathrm{C}$. Необходимая температура термообработки достигалась в течение $1 \mathrm{~h}$, после чего при данной температуре они удерживались разное время. Образец $T_{1}$ не подвергался термообработке, образцы $T_{2}, T_{3}, T_{4}, \ldots, T_{7}$ отжигались при $530^{\circ} \mathrm{C}$ соответственно $t_{2}=15, t_{3}=35$, $t_{4}=65, t_{5}=125, t_{6}=245, t_{7}=425 \mathrm{~min}$. Образец $A_{1}$ не подвергался термообработке, образцы $A_{2}, A_{3}, A_{4}, \ldots A_{7}$ отжигались при $500^{\circ} \mathrm{C}$ соответственно $t_{2}=10, t_{3}=15$, $t_{4}=30, t_{5}=80, t_{6}=120, t_{7}=180, t_{8}=270, t_{9}=420$, $t_{10}=510, t_{11}=630, t_{12}=810, t_{1} 3=1050, t_{14}=1410$, $t_{15}=1890 \mathrm{~min}$.

Спектры оптического пропускания нанокристаллов $\mathrm{CdSe}_{x} \mathrm{~S}_{1-x}$ в силикатном стекле в диапазоне 350-700 nm исследовались с помощью спектрометра USB-4000(Ocean optics Inc.)

Исследование спектров оптического поглощения полупроводниковых материалов, кристаллическая решетка которых содержит структурные дефекты, является эффективным методом, который позволяет найти корреляцию между структурой и оптическими свойствами изучаемого материала. В настоящей работе исследование оптических спектров пропускания образцов, которые находятся в разных стадиях формирования нанокристаллов, позволяет понять поведение структурных дефектов в этих нанокристаллах.

На рис. 1 приведены спектральные зависимости коэффициента поглощения $(\alpha=f(h v))$ для некоторых образцов. Как видно, на графиках зависимости $\alpha=f(h v)$ можно выделить два участка. На участках, где поглощение слабое, зависимость $\alpha=f(h v)$ имеет экспоненциальный характер. Этот участок хорошо известен 


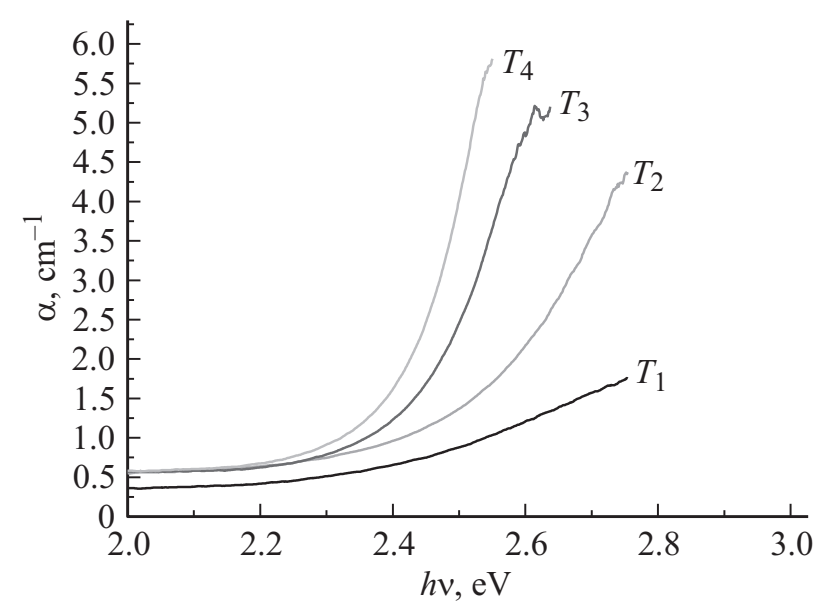

Pис. 1. Спектральные зависимости коэффициента поглощения образцов серии $T$.

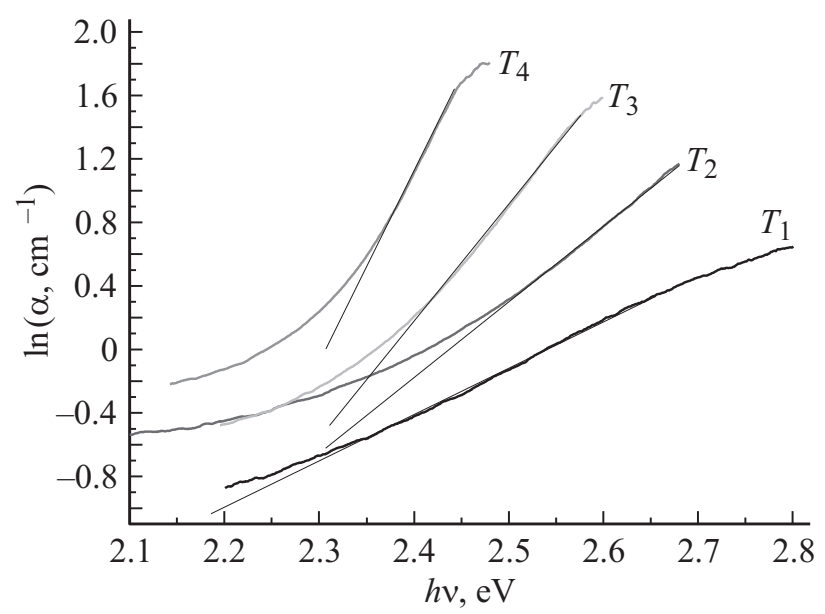

Рис. 2. Зависимость $\ln \alpha$ от энергии падающего фотона.

под названием „хвост Урбаха“, и он наблюдается в большинстве некристаллических (аморфных) твердых тел [15]. Самое распространенное объяснение экспоненциальной зависимости $\alpha=f(h v)$ связывают с наличием неупорядоченности на атомном уровне в исследуемых структурах, т.е. с контенуальным нарушением ближнего порядка [16]. Под последним подразумеваются пространственные флуктуации величин межатомных расстояний и углов между направлениями на различные атомы. В экспоненциальном участке зависимость $\alpha=f(h v)$ подчиняется правилу Урбаха [15]:

$$
\alpha=\alpha_{0} \exp \frac{-\left(E_{g}-h v\right)}{E_{0}}
$$

где $E_{g}$ - оптическая ширина запрещенной зоны, $h v-$ энергия кванта падающего света, $E_{0}-$ характеристическая энергия, которая определяет крутизну края и несет информацию о среднеквадратичном отклонении межатомных расстояний. Используя модель случайного поля, созданного хаотически распределенными в про- странстве заряженными атомами, для характеристической энергии в работе [17] была получена формула.

$$
E_{0}=2.2 W_{B}\left(N_{t} a_{B}^{3}\right)^{2 / 5},
$$

где $W_{B}=e^{2} / 2 \varepsilon a_{B}, a_{B}-$ боровский радиус в веществе, $\varepsilon$ - диэлектрическая проницаемость, $N_{t}$ - эффективная концентрация заряженных центров.

Для того чтобы определить характеристическую энергию $E_{0}$, на графиках спектральной зависимости коэффициента поглощения $\alpha=f(h v)$ мы выделили участки экспоненциальной зависимости. Для этого были построены зависимости $\ln \alpha$ от $h v$ и выбраны те участки, где эти зависимости становятся линейными. Несколько примеров таких зависимостей приведены на рис. 2. Характеристическая энергия $E_{0}$ определялась обратной величиной крутизны линейных участков.

На рис. 3 показаны зависимости $E_{0}$ от длительности термообработки при температурах 500 и $530^{\circ} \mathrm{C}$ для образцов серии $A$ и $T$. Как видно, значение $E_{0}$ сначала падает довольно резко, а потом более медленно. Кроме того, чем выше температура термообработки, тем резче падает значение $E_{0}$. Имея значение $E_{0}$, с помощью выражения (2) можно было определить зависимость
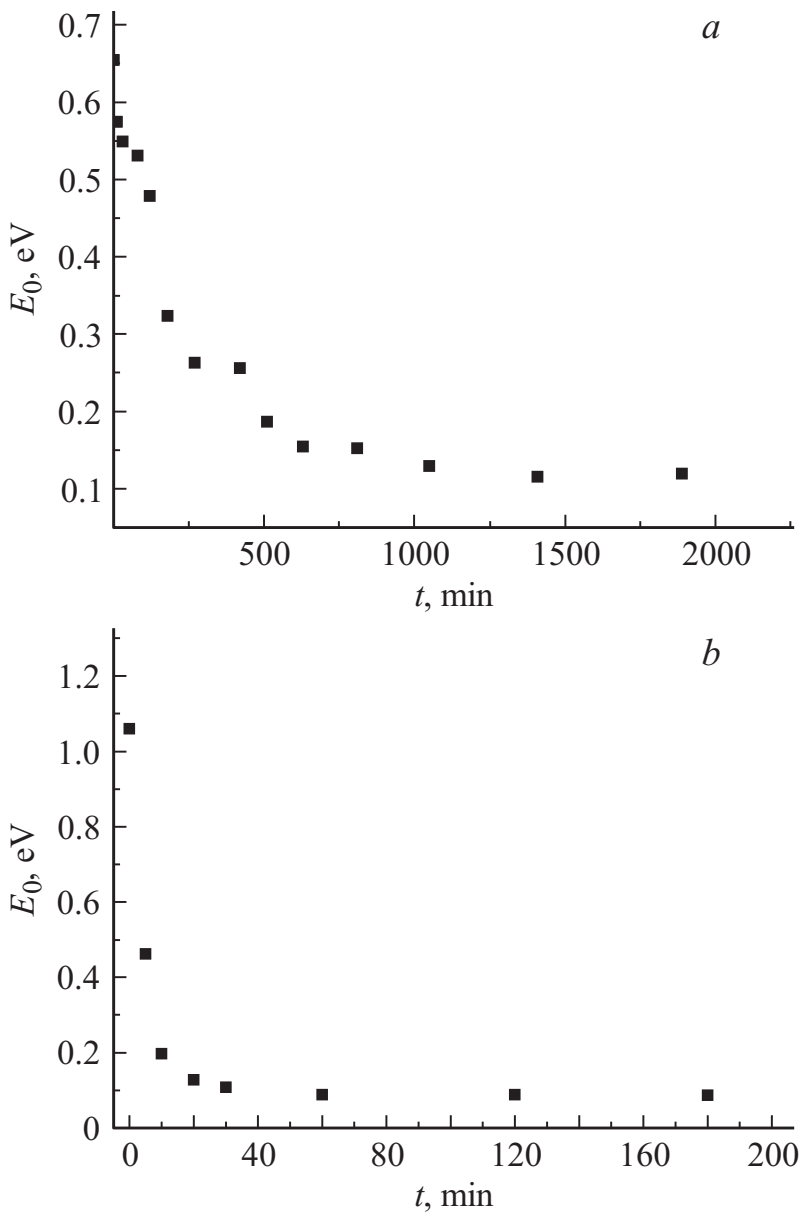

Рис. 3. Зависимость характеристической энергии $E_{0}$ от времени термообработки при $t,{ }^{\circ} \mathrm{C}: a-500, b-530$. 


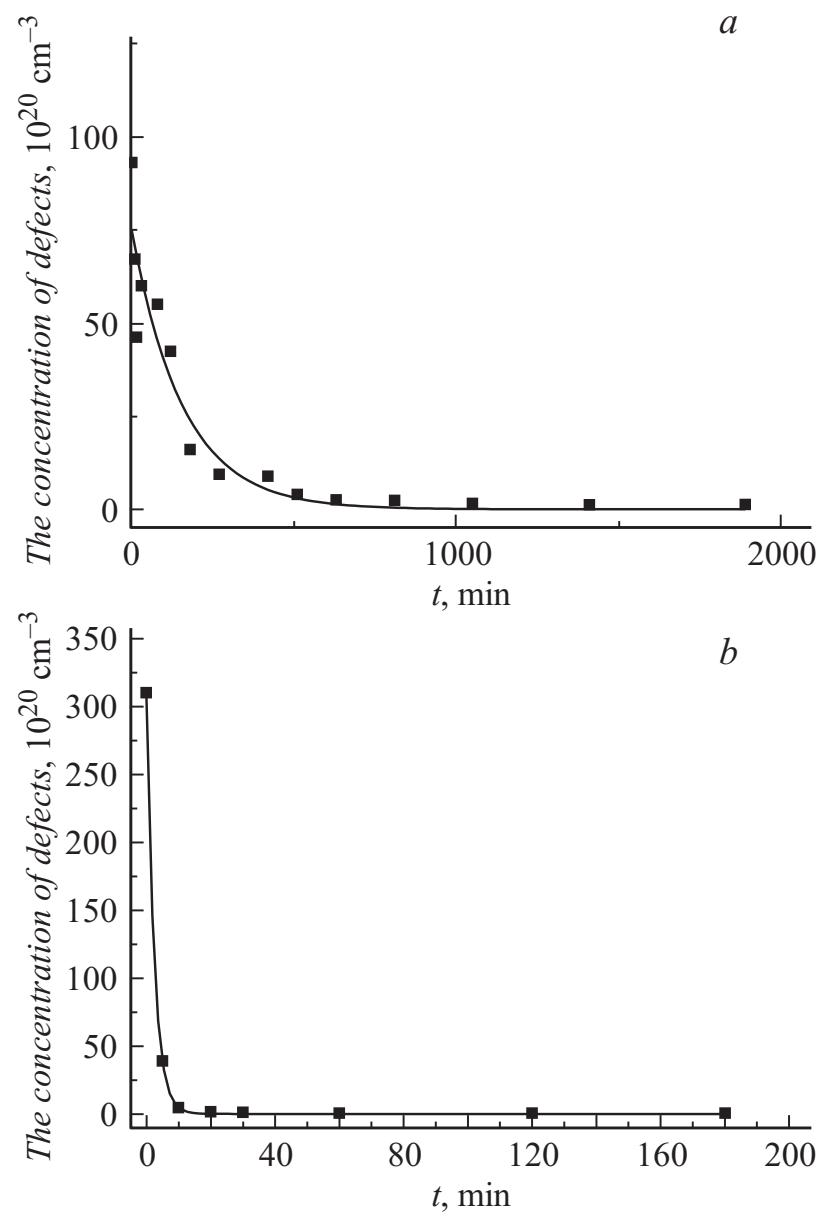

Рис. 4. Зависимость эффективной концентрации заряженных центров от времени термообработки при $t,{ }^{\circ} \mathrm{C}: a-500, b-$ 530.

эффективной концентрации дефектов от длительности термообработки при температурах 500 и $530^{\circ} \mathrm{C}$ (рис. 4). Зависимость эффективной концентрации дефектов от длительности термообработки хорошо описывается формулой $N=N_{0} \exp (-t / \tau)$, где $N_{0}-$ концентрация дефектов до термообработки, а $\tau$ назовем временем релаксации дефектов. При термообработке $500^{\circ} \mathrm{C}$ время релаксации составило $158 \mathrm{~min}$, а при $530^{\circ} \mathrm{C}-2.4 \mathrm{~min}$. Такая сильная зависимость времени релаксации дефектов от температуры термообработки говорит о том, что процесс восстановления дефектов носит активационный диффузионный характер. Связь времени релаксации с энергией активации диффузии в таких случаях можно представить следующим выражением:

$$
\tau=\tau_{0} \exp \left(\frac{E_{\mathrm{diff}}}{k T}\right)
$$

где $E_{\text {diff }}-$ энергия активации диффузии. Исходя из значения времени релаксации, было оценено значение энергии активации молекул $\mathrm{CdSe}_{x} \mathrm{~S}_{1-x}$, растворенных в силикатном стекле. Оно составляло приблизительно $8 \mathrm{eV}$.
Наличие дефектов в нанокристаллах с энергетической точки зрения эквивалентно возникновению дискретных уровней в запрещенной зоне. Поглощение на этих уровнях и является причиной возникновения хвоста Урбаха. Уменьшение концентрации дефектов в процессе термообработки должно проявляться в спектрах поглощения. С уменьшением концентрации кристаллическая решетка нанокристаллов становится более совершенной, уменьшается концентрация дискретных уровней в запрещенной зоне, и край поглощения должен стать более резким, что и должно привести к уменьшению ширины хвоста Урбаха.

На рис. 5 показны зависимости ширины экспоненциальной области от времени термообработки для образцов серии $T$ и $A$. Полученные зависимости подтверждают наше предположение, более того, чем выше температура термообработки, тем быстрее происходит формирование кристаллической решетки в нанокристаллах.

В спектральной зависимости коэффициента поглощения можно выделить вторую область - область фундаментального поглощения, которая соответствует электронным переходам зона-зона. Для определения ширины запрещенной зоны используется область фунда-
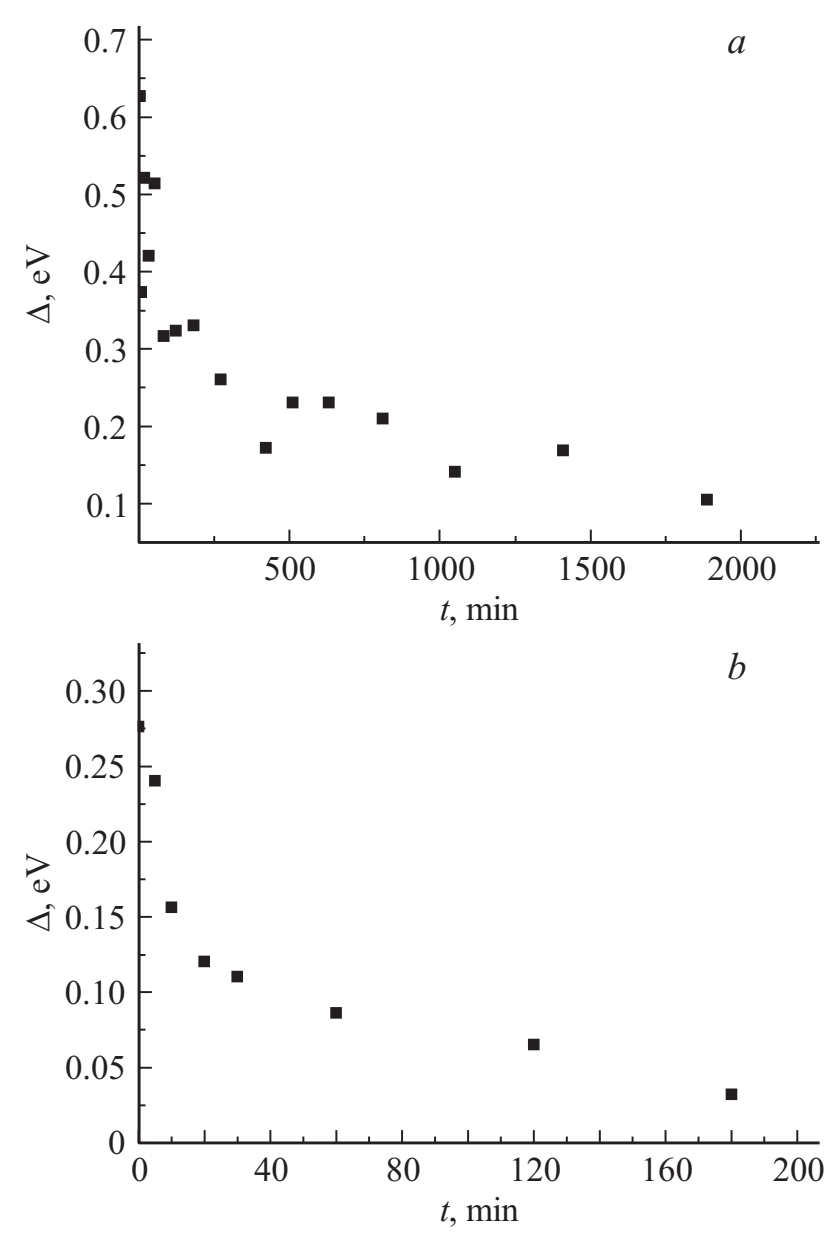

Рис. 5. Зависимость ширины области экспоненциальной зависимости от времени термообработки при $t,{ }^{\circ} \mathrm{C}: a-500$, $b-530$. 


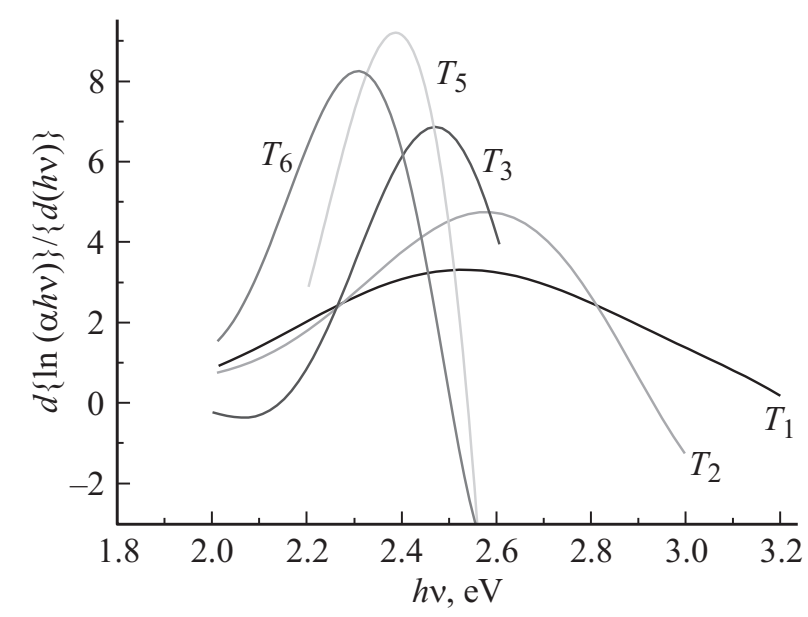

Рис. 6. Зависимость $\frac{d\{\ln (\alpha h v)\}}{d(h v)}$ от энергии падающего фотона.

ментального поглощения. В данной области спектральная зависимость коэффициента поглощения определяется формулой Таuс-а [18]

$$
(\alpha h v)^{1 / m}=A\left(h v-E_{g}\right),
$$

где $\alpha-$ коэффициент поглощения, $h v-$ энергия подающего фотона, $A-$ постоянная, зависящая от природы оптических переходов, $E_{g}$ - оптическая ширина запрещенной зоны, $m$ - величина, значение которой определяется типом перехода, который в свою очередь зависит от структуры материала. Для массивных полупроводников $m$ принимает значения $1 / 2,2,3 / 2$ и 3 , которые соответствуют разрешенным прямым, разрешенным непрямым, неразрешенным прямым и неразрешенным непрямым переходам [19]. Поскольку в нашем случае исследуемый полупроводниковый материал имеет прямозонную структуру, доминирующими переходами должны быть разрешенные прямые переходы, что соответствует значению $m=1 / 2$.

Для определения ширины запрещенной зоны $\left(E_{g} 1\right)$ был построен график зависимости $(\alpha h v)^{2}$ от $h v$. Значение энергии запрещенной зоны определялось экстраполяцией прямолинейного участка графика в сторону оси энергии. У образцов, которые находятся в начальной стадии образования, не всегда возможно выделить линейные участки на графиках зависимости $(\alpha h v)^{2}=f(h v)$, что усложняет определение $E_{g}$. Однако значение $E_{g}$ можно определить с помощью зависимости $d\{\ln (h v)\} / d(h v)$ от $h v$. Действительно, из формулы (4) видно, что

$$
\frac{d\{\ln (\alpha h v)\}}{d(h v)}=\frac{m}{\left(h v-E_{g}\right)} .
$$

Теоретически зависимость $d\{\ln (h v)\} / d(h v)$ от $h v$ при $h v=E_{g}$ должна иметь разрыв. А в действительности за значение энергии запрещенной зоны $\left(E_{g} 2\right)$ можно принимать то значение $h v$, при котором упомянутая зависимость принимает максимальное значение. Для того чтобы построить график $d\{\ln (h v)\} / d(h v)$ от $h v$, сначала экспериментально полученная зависимость $\ln (\alpha h v)=f(h v)$ с весьма высокой точностью была аппроксимирована многочленом девятой степени, по которому была вычислена $d\{\ln (h v)\} / d(h v)$ и построен график зависимости $d\{\ln (h v)\} / d(h v)$ от $h v$. На рис. 6 приведены несколько примеров зависимости $d\{\ln (h v)\} / d(h v)$ от $h v$. Как видно из этих зависимостей, на начальном этапе образования нанокристаллов максимумы слабо выражены. Они становятся резкими в процессе термообработки, что свидетельствует об уменьшении концентрации дефектов при термообработке и, следовательно, поглощения, связанного с этими дефектами.

На рис. 7 показаны зависимости оптической ширины запрещенной зоны от времени термообработки, которые были получены двумя разными способами. Как видно, эти значения $E_{g}$ достаточно близки.

Когда размеры нанокристаллов становятся сопоставимы с радиусом Бора для электрона, величина оптической ширины запрещенной зоны определяется формулой [20]

$$
E_{g}=E_{g 0}+\frac{h^{2} \pi^{2}}{2 \mu R^{2}},
$$

где $E_{g 0}$ - ширина запрещенной зоны массивного образца, $\mu=\frac{m_{n} m_{p}}{m_{n}+m_{p}}, R-$ средний радиус квантовой точки. Уменышение энергии запрещенной зоны от времени термообработки объясняется увеличением размеров нанокристаллов.

Отметим, что зависимость коэффициента $m$ в формуле (4) от $h v$ содержит важную информацию об изменении природы оптических переходов в нанокристаллах. Значение $m$ определялось как крутизна зависимости $\ln (\alpha h v)=f\left(\ln \left(h v-E_{g}\right)\right.$, так как, согласно формуле (4),

$$
\ln (\alpha h v)=\ln A+m \ln \left(h v-E_{g}\right) .
$$

Для получения спектральной зависимости коэффициента $m$ на основе ранее полученных экспериментальных результатов был построен график зависимости

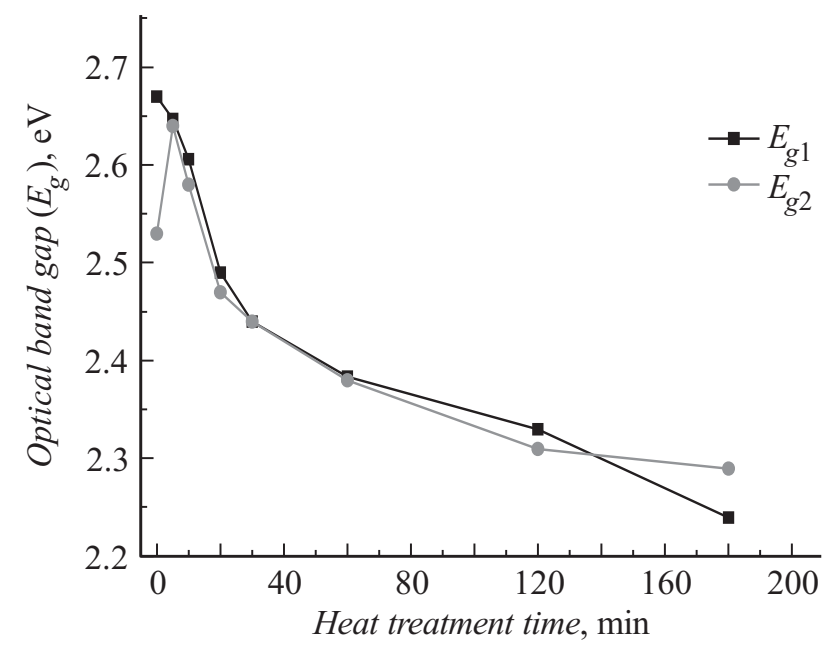

Рис. 7. Зависимость оптической ширины запрещенной зоны от времени термообработки. 


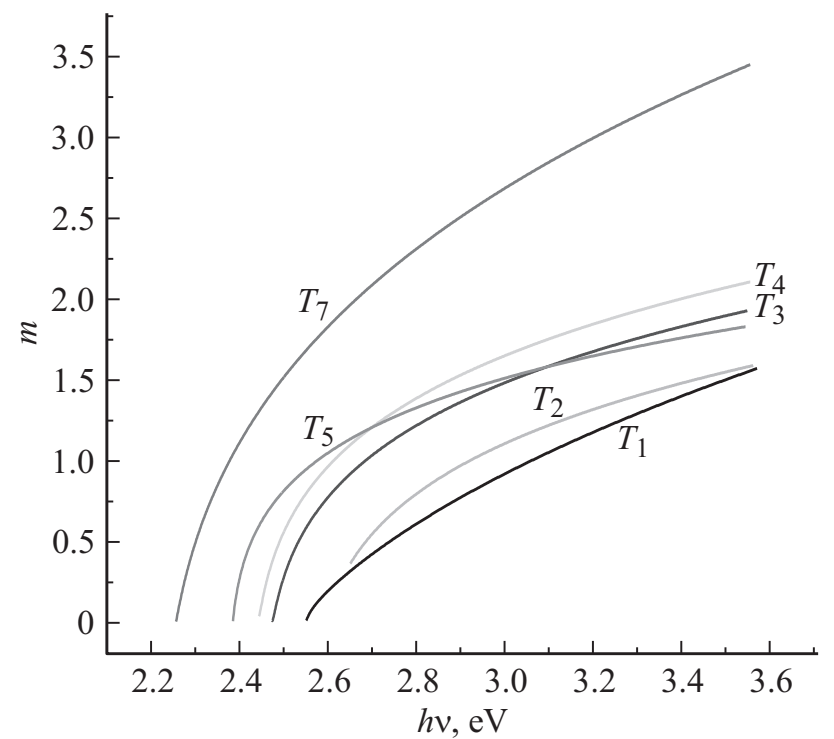

Рис. 8. Зависимость коэффициента $m$ от энергии падающего фотона.

$\ln (\alpha h v)=f\left(\ln \left(h v-E_{g}\right)\right.$. Полученная зависимость была аппроксимирована с достаточно высокой точностью многочленом четвертой степени. Значение коэффициента $m$ определялось как производная полученного математического выражения $d\{\ln (\alpha h v)\} / d\left\{\left(h v-E_{g}\right)\right\}$.

На рис. 8 приведены спектральные зависимости коэффициента $m$ для образцов серии $T$ в области сильного поглощения. Как видно, значение $m$ монотонно растет с ростом $h v$ и меняется в диапазоне (0-3). Такое изменение значения $m$ говорит о том, что в зависимости от энергии подающего фотона может измениться весовой вклад различных типов оптических переходов. Если на начальном этапе образования нанокристаллов значение $m$ в диапазоне бо́льших значений $h v$ изменяется в непосредственной близости $m=0.5$, что соответствует разрешенным прямым переходам, то в дальнейшем значение $m$ приближается к $3 / 2$, что соответствует прямым неразрешенным переходам. Для получения закона изменения $m$ от $h v$ полученные экспериментальные зависимости были аппроксимированы формулой $m=K\left(h v-E_{g}\right)^{c}$. Этим выражением достаточно хорошо описываются экспериментально полученные зависимости $m=f(h v)$ для всех образцов, а значение „,“ от образца к образцу изменяется в диапазоне $0.36-0.7$.

\section{Заключение}

Таким образом, исследования спектральной зависимости коэффициента поглощения нанокристаллов $\mathrm{CdSe}_{x} \mathrm{~S}_{1-x}$ в силикатном стекле показали, что в области $h v<E_{g}$ она подчиняется закону Урбаха, на основе которого было оценено значение характеристической энергии $E_{0}$, которая несет информацию о среднеквадратичном отклонении межатомных расстояний для об- разцов с разной длительностью термообработки. С использованием модели случайного потенциала, который возникает из-за хаотичного распределения атомов, была получена зависимость концентрации дефектов от времени термообработки.

Показано, что процесс исчезновения дефектов носит тепловой активационный характер. Было также оценено значение энергии активации диффузии.

\section{Список литературы}

[1] Alferov Zh. // Rev. Mod. Phys. 2001. Vol. 73. N 3. P. 767.

[2] Асрян Л.В., Сурис Р.С. // ФТП. 2004. Т. 38. Вып. 1. С. 3-26.

[3] Peng X., Manna L., Yang W., Wickham J., Scher E., Kadavanich A., Alivisatos A.P. // Nature. 2000. Vol. 404. P. 59.

[4] Kim L.Y., Jung H.J., Kim K.H., Yoon S.H., Song S.M., Bae H.S., Kim Y. // Nanotechnology. 2009. Vol. 20. P. 095605.

[5] Masumoto Y., Takagahara T. // Semiconductor Quantum Dots. Berlin: Springer. 2002.

[6] Chan W., Nie S. // Science. 1998. Vol. 281. P. 2016.

[7] Dabbousi B.O., Bawendi M.G., Onotsuka O., Rubner M.F. // Appl. Phys. Cem. B.1995. Vol. 66. P. 1316.

[8] Gratzel M. // Nature. 2001. Vol. 414. P. 338.

[9] Moris D., Egdell R.J. // Mater. Chem. 2001. Vol. 11. P. 3207.

[10] Бондарь И.В., Гурин В.С., Соловей Н.П., Молочко А.П. // ФТП. 2007. Т. 41. Вып. 8. С. 959-965.

[11] Ekimov A.I., Efros A.L. // Phys. Stat. Sol. B. 1988. Vol. 150. P. $627-631$.

[12] Суздалев И.П. Нанотехнология: физико-химия нанокластеров, наноструктур и наноматериалов. М.: Ком. книга, 2006.

[13] Grigoryan L., Petrosyan P., Petrosyan S., Bellani V., Maglia F. // Eur. Phys. J. 2003. Vol. 34. P. 415-419.

[14] Седракян Д.М., Петросян П.Г., Григорян Л.Н // ЖТФ. 2015. Т. 85. Вып. 5. С. 94-99.

[15] Urbach F. // Phys. Rev. 1953. Vol. 92. P. 1324.

[16] Клява Г.Я. // ФТТ. 1985. Т. 27. Вып. 5. С. 1350-1353.

[17] Бонч-Бруевич В.Л. // УФН. 1983. Т. 140. С. 583.

[18] Pankov J.I. // Optical Processes in Semiconductors. New Jersey, USA. 1971. P. 34.

[19] Bhattacharjee B., Hsu C.H., Lu C.H., Ruaan R.C. // Chalcogen. Lett. 2010. Vol. 7. P. 71-81.

[20] Kayanuma Y. // Phys. Rev. 1988. Vol. 38. P. 9797. 